\title{
Ciliary body melanoma with optic nerve invasion
}

\author{
Saad Al-Haddab, Ahmed Hidayat, Khalid F Tabbara
}

\begin{abstract}
A case of melanoma of the ciliary body is presented. Initially the patient was diagnosed and treated for uveitis, but following CT scanning and ultrasound a tumour was detected and the eye enucleated. Histopathologically it was found that the tumour had invaded the optic nerve head, apparently via Cloquet's canal.
\end{abstract}

Malignant melanomas of the ciliary body are rare. ${ }^{1-4}$ In contrast to retinoblastoma, invasion of the optic nerve by uveal melanoma is also rare. ${ }^{3}$ This condition was noted in $3.6 \%$ of the cases summarised by Reese. ${ }^{1}$

We report herewith a case of ciliary body melanoma that masqueraded as anterior uveitis and invaded the optic nerve.

\section{Case report}

A 20-year-old man presented with a history of decrease of vision in his left eye of two months' duration. He had no history of previous medical or ocular problems. Elsewhere he had been diagnosed as having uveitis. Topical steroids were prescribed, which consisted of prednisolone acetate $1 \%$ every two hours. The right eye showed nothing abnormal. The left eye showed a visual acuity of no light perception. Intraocular tension in the left eye was $45 \mathrm{mmHg}$ by applanation tonometry. Pigmented precipitates were seen on the lower quadrants of the corneal endothelium, and there were $2+$ cells and a $2+$

\footnotetext{
Department of

Ophthalmology, College of Medicine, King Saud University, and the King Khaled Eye Specialist Hospital, Riyadh, Saudi Arabia; and Department of Ophthalmic Pathology, Armed Forces Institute of Pathology, Washington, DC, USA

S Al-Haddab A Hidayat

K F Tabbara

Correspondence to K F Tabbara, MD Department of Ophthalmology, PO Box 55307, Riyadh 11534 , Saudi Arabia.

Accepted for publication 23 August 1989
}

Figure 1: The upper and lower halves of a composite computerised tomography scan of the left orbit showing the ciliary body mass nasally. The lower half shows slight thickening of the left optic nerve in comparison with the right. flare reaction in the anterior chamber. Small, round, yellowish nodules on the iris were seen, with rubeosis at the $3 o^{\prime}$ 'clock position. The pupil was irregular, with a $360^{\circ}$ posterior synechia. The lens was cataractous and had pigmentary deposits over its anterior surface. The fundus could not be seen.

A computerised tomographic scan of the left orbit showed a large mass located on the ciliary body nasally, with evidence of choroidal excavation (Fig 1) and thickening of the optic nerve. Ultrasound examination of the left eye showed a large, moderately reflective, slightly irregular mass located at the ciliary body nasally. The patient underwent enucleation of the left eye. Six months after the enucleation the patient had a clear socket without any evidence of recurrence of the melanoma in the orbit.

\section{PATHOLOGICAL FINDINGS}

Grossly a large black ciliary body tumour was located nasally; it measured $10 \times 10 \mathrm{~mm}$ at its base and $6 \mathrm{~mm}$ in height. Anteriorly there was seeding into the anterior chamber angle and on to the posterior surface of the cornea. Posteriorly there was seeding into the vitreous body behind the lens and into Cloquet's canal in the form of a brown strand that blended posteriorly with a small brown mass covering and obscuring the optic nerve head (Fig 2). The $6 \mathrm{~mm}$ optic nerve stump was thickened measuring $5.5 \mathrm{~mm}$ in thickness. A cross-section of the optic nerve at the line of surgical transection showed brown discoloration. Microscopically there was a large, heavily pigmented, mostly necrotic, malignant melanoma that involved the ciliary body and a small portion of the adjacent peripheral choroid (Fig 3).

The neoplasm had also invaded the trabecular meshwork, canal of Schlemm, and the collector channels. The small area at the level of pars

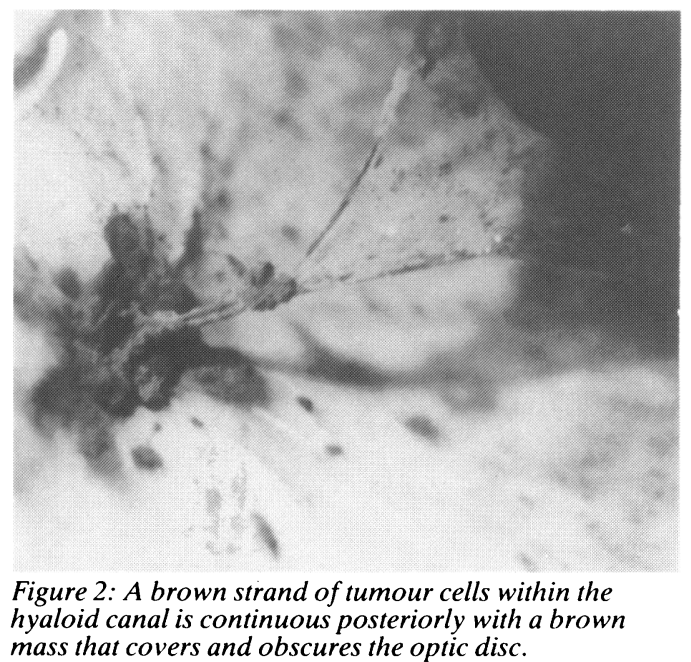




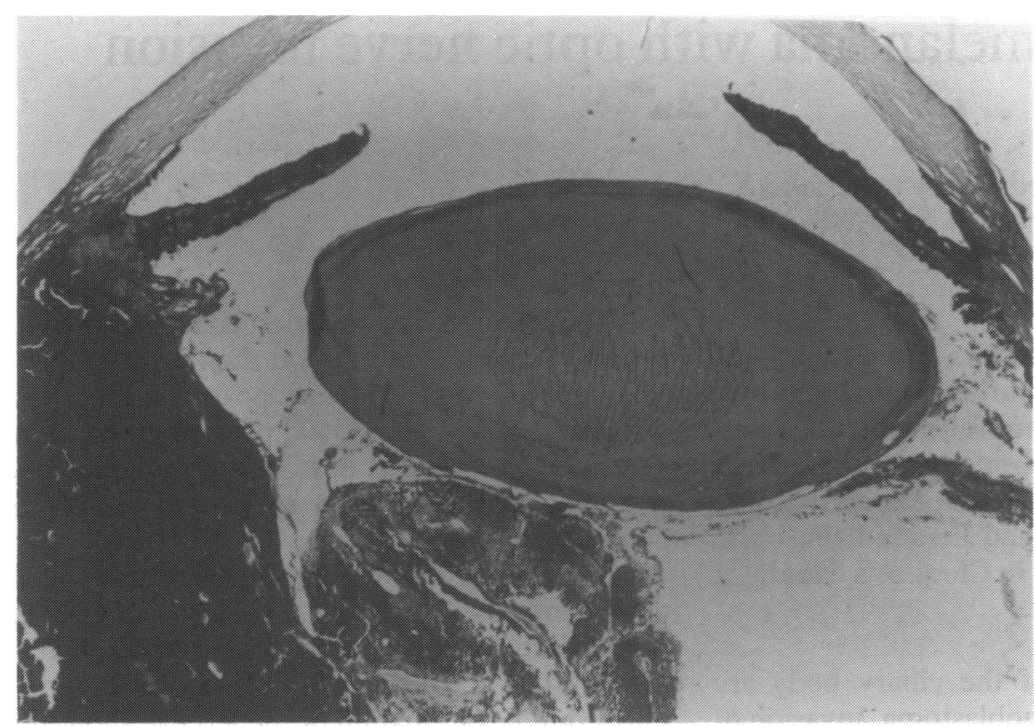

Figure 3: The large and heavily pigmented ciliary body melanoma is associated with seeding of tumour cells into the vitreous, posterior chamber, and anterior chamber angle. plana, where viable cells were present showed a mixture of epithelioid and spindle B cells.

\section{Discussion}

This case of ciliary body melanoma masqueraded as anterior uveitis. The large necrotic area of the tumour is believed to have induced inflammatory response and uveitis. Although retinoblastomas commonly invade the optic nerve, extension of uveal melanoma to the optic nerve is rare. In our patient the tumour cells reached the optic nerve via Cloquet's canal, vitreous body, and retina. Thickening of the left optic nerve was noted by computerised tomography in comparison with the sound eye. Extension of uveal melanoma to the optic nerve occurred in $3.7 \%$ of 432 cases reviewed by Shammas and Blodi. ${ }^{2}$ The optic nerve can be invaded by a uveal melanoma by direct extension through the vitreous and shedding of tumour cells. ${ }^{34}$

A number of factors have been noted to increase the likelihood of extension to the optic nerve. They include raised intraocular pressure, large tumour size, diffuse growth pattern, epithelioid cell type, tumour necrosis, glaucoma, and peripapillary location. ${ }^{2-4}$ Another important factor which made the prognosis worse in our patient was invasion of the collecting channels.

1 Reese AB. Tumors of the eye. 2nd ed. New York: Harper and Row, 1963: 265-92.

2 Shammas HF, Blodi FC. Peripapillary choroidal melanomas. Arch Ophthalmol 1978; 96: 440-5.

3 Spencer WH. Optic nerve extension of intraocular neoplasms. Am 7 Ophthalmol 1975; 80: 465-71.

4 Spencer WH, Inverson HA. Diffuse melanoma of the iris with extrabulbar extension via the optic nerve. Surv Ophthalmol 1965; 10: 365-71. 